\title{
Umbilical cord: an allogenic tissue for potential treatment of COVID-19
}

\author{
Hugo C. Rodriguez ${ }^{1,2,3,4} \cdot$ Manu Gupta $^{1}$ - Emilio Cavazos-Escobar ${ }^{2,5} \cdot$ Saadiq F. El-Amin III ${ }^{6,7}$. Ashim Gupta ${ }^{1,4,7,8}$ (D)
}

Received: 6 September 2020 / Accepted: 30 September 2020 / Published online: 9 October 2020

(c) Japan Human Cell Society 2020

\begin{abstract}
The COVID-19 pandemic has placed an unprecedented burden on health care systems and economies around the globe. Clinical evidences demonstrate that SARS-CoV-2 infection produces detrimental levels of pro-inflammatory cytokines and chemokines that can lead to acute respiratory distress syndrome (ARDS) and significant systemic organ damage. Currently, there is no definitive therapy for COVID-19 or associated complications, and with the hope of a safe and effective vaccine in the distant future, the search for an answer is paramount. Mesenchymal stem cells (MSCs) provide a viable option due to their immunomodulatory effects and tissue repair and regeneration abilities. Studies have demonstrated that compassionate use of MSCs can reduce symptoms associated with SARS-CoV-2 infection, eliminate fluid buildup, and act as a regenerative technique for alveolar damage; all in a safe and effective way. With multiple autologous sources available for MSCs, each with their own respective limitations, allogenic umbilical cord (UC) and/or UC-derived Wharton's jelly (WJ) seem to be best positioned source to harvest MSCs to treat COVID-19 and associated symptoms. As an allogenic source, UC is readily available, easily obtainable, and is rich in immunomodulatory and regenerative factors. In this manuscript, we reviewed the current evidences and explored the potential therapeutic use of allogenic UC and/or WJ-derived MSCs for the treatment of COVID-19. Although, preliminary preclinical and clinical studies indicate that their use is safe and potentially effective, more multi-center, randomized, controlled trials are needed to adequately assess the safety and efficacy of UC and/or WJderived MSCs for the treatment of COVID-19.
\end{abstract}

Keywords Umbilical cord · Wharton's jelly · Mesenchymal stem cells · COVID-19 · Coronavirus

\section{Background}

For over 50 years, human coronaviruses (HCoVs) have been reported as causes of mild to severe respiratory infections in humans [1]. HCoVs subtypes such as HCoV-299E and HcoV-OC43 are known to cause mild respiratory symptoms in healthy adults while HCoVs such as severe acute

Ashim Gupta

ashim6786@gmail.com

Hugo C. Rodriguez

hcrodrig@student.uiwtx.edu

Manu Gupta

manu6771@yahoo.co.in

Emilio Cavazos-Escobar

emicavaz@utmb.edu

Saadiq F. El-Amin III

dr.saadiqelamin@gmail.com

1 Future Biologics, 1110 Ballpark Ln Apt 5109, Lawrenceville, GA 30043, USA respiratory syndrome coronavirus (SARS) and Middle East respiratory syndrome coronavirus (MERS) have shown higher pathogenicity and are known causes of global outbreaks [1, 2]. In December 2019 in a wholesale wet market in Wuhan, Hubei, China a novel $\mathrm{HCoV}$ (severe acute respiratory syndrome coronavirus 2) began its introduction to the world [3]. Severe acute respiratory syndrome coronavirus 2

2 Future Physicians of South Texas, San Antonio, TX, USA

3 School of Osteopathic Medicine, University of the Incarnate Word, San Antonio, TX, USA

4 South Texas Orthopaedic Research Institute, Laredo, TX, USA

5 University of Texas Medical Branch at Galveston, Galveston, TX, USA

6 El-Amin Orthopaedic and Sports Medicine Institute, Lawrenceville, GA, USA

7 BioIntegrate, Lawrenceville, GA, USA

8 Veterans in Pain, Los Angeles, CA, USA 
(SARS-CoV-2) started its spread across China and surrounding countries (Thailand, Republic of Korea and Japan) eventually leading to a global pandemic [3]. As of September 6,2020 , there are about 27 million reported cases and over 881,000 deaths worldwide, with over 6.26 million reported cases and over 188,000 deaths in the United States [4]. With no current vaccine or definitive therapy for SARS-CoV-2 or its disease (coronavirus disease 2019), there is an obvious urgent need for safe and efficacious treatment modalities.

Currently, the treatment of coronavirus disease 2019 (COVID-19) centers around a broad range of pharmaceuticals (chloroquine/hydroxychloroquine, glucocorticoids and antivirals), convalescent plasma transfusions and respiratory aid (noninvasive and invasive respiratory therapy) [5, 6]. With none of the available treatment modalities showing definitive effectiveness and the resulting strain on the hospital resources, mesenchymal stem cells (MSCs) present a promising option to combat COVID-19 and the present global pandemic [7, 8]. MSCs have been shown to be a potentially effective and safe treatment modality for inflammatory lung diseases with a large number of preclinical studies showing positive immunomodulatory and regenerative effects [9]. For this reason, there has been increased effort placed into developing clinical trials using MSCs in COVID-19 patients, with a large number of current ongoing trials. MSCs when intravenously administered have shown to accumulate within the lung microvasculature, promoting endogenous repair of local cells, protecting alveolar epithelial cells and decreasing lung fibrosis [10]. MSCs have also been shown to decrease the hyperactivated immune system and subsequent elevated cytokine and chemokine levels reported in COVID-19 patients [11]. MSCs are able to inhibit T-lymphocyte proliferation, B-lymphocyte proliferation and macrophage activation leading to decreased levels of proinflammatory cytokines such as interleukin-6 (IL-6) and tumor necrosis factor alpha (TNF- $\alpha$ ), while simultaneously increasing IL-10, an essential anti-inflammatory cytokine $[11,12]$.

There are various sources of MSCs such as autologous bone marrow, adipose tissue, dental pulp (DP), umbilical cord (UC) and/or UC-derived Wharton's jelly (WJ) [10, 13, 14]. Autologous bone marrow requires the patient to undergo an invasive procedure associated with pain and morbidity to harvest the MSCs [15]. The number of MSCs in the bone marrow (bone marrow mesenchymal stem cells, and BMSCs) is limited (0.001-0.01\%) and are also known to display characteristics of early senescence [15]. Adiposederived stem cells (ADSCs) harvest also requires the patient to undergo an invasive procedure and are considered to be in the preliminary steps of clinical investigation requiring more randomized clinical trials to prove their efficacy and safety [16]. DP MSCs have several limitations as well due to their limited availability in adult humans and lack of regeneration after extraction [17]. The UC is a neonatal tissue that is rich in easily expandable and accessible MSCs [10]. The UC stroma otherwise known as WJ is an ample source of MSCs and is also widely used to harvest MSCs [18]. WJ is a connective tissue located within the UC known to resist compressive and torsional forces during fetal development. WJ contains high levels of primitive MSCs with the highest concentration of MSCs per milliliter than any other allogenic tissue [19]. WJ-MSCs have also been shown to be mostly multipotent rather than pluripotent and secrete large quantities of anti-inflammatory cytokines (CKs), growth factors (GFs) and extracellular vesicles (EVs) including exosomes compared to other sources [18, 20,21]. With all these factors considered, the UC and/or WJ is an ideal allogenic source of MSCs for treating COVID-19 patients and decreasing the severe strain on hospital resources.

\section{Pathophysiology of SARS-CoV-2 infection}

Understanding the pathophysiology of SARS-CoV-2 is imperative if novel treatments are to be properly investigated. These viruses are enveloped, positive sense, singlestranded RNA viruses of about 30 kilobases and are transmitted in infected air droplets that enter the human body through the nose, mouth, or eyes [22, 23]. The virus then attaches to its functional receptor, angiotensin converting enzyme 2 (ACE2), via the spike (S) protein expressed on the surface of the virus [8]. RNA is then released into the host cell that travels to the nucleus for replication [10,22, 24]. It is important to note that ACE 2 receptors are widely expressed in epithelial cells not only of the lungs but also the heart, liver, kidneys and digestive organs [12]. Once the virus enters the bloodstream it spreads widely throughout the body [12]. One of the major barriers faced by scientists and clinicians is the diverse manifestation of the disease among patients. Some patients present with severe symptoms while others can be infected but appear asymptomatic [25]. There is a common pattern of disease progression in which the symptoms appear about 2-14 days after viral exposure which include fever, muscle pain, headache, cough, sore throat, and loss of taste or smell [25]. The patients requiring intensive care unit (ICU) treatment are commonly older male patients with prominent comorbidities such as obesity, hypertension, cardiovascular disease, and lung disease, many of which are highly prevalent $[26,27]$.

One of the most popular theories for the cause of SARSCoV-2-related complications is due to the body's own immune response. An overactivated immune system has the potential to kill the virus, but also produce a large number of pro-inflammatory factors, leading to the severe hypercytokinemia, also known as a cytokine storm (Fig. 1) [26]. Once a cell is infected, cytokines (e.g., IL-6, IL-8, IL-10, 


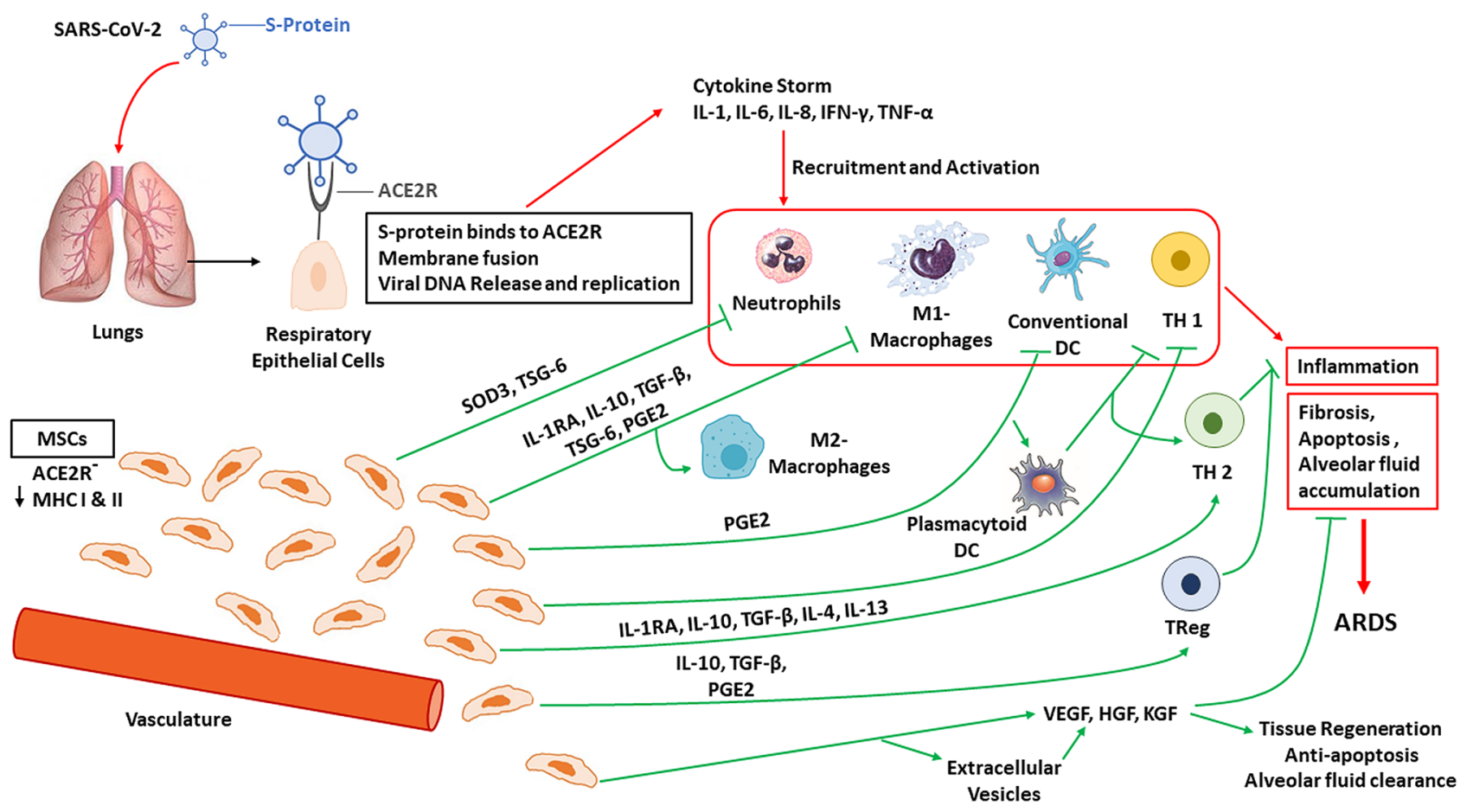

Fig. 1 SARS-CoV-2 induced cytokine storm and development of ARDS. When SARS-CoV-2 enters the lungs, it's S-protein binds to the ACE2R on respiratory epithelial cells and leads to membrane fusion, viral DNA release and replication inducing a cytokine storm. This cytokine storm leads to the recruitment and activation of neutrophils, M1 macrophages, conventional DC and TH $1 \mathrm{~T}$ cells, all of which induces inflammation, fibrosis, apoptosis and alveolar fluid accumulation within the lungs leading to ARDS. Therapeutic intravenous administration of MSCs lead to accumulation of MSCs in the lung microvasculature and inhibit inflammation by shifting the M1 macrophage, conventional DC, and TH 1 T-cell populations into antiinflammatory M2 macrophage, plasmacytoid DC, TH 2/TReg T-cell populations as well as directly inhibiting neutrophils. Additionally, MSC therapy inhibits fibrosis, apoptosis and alveolar fluid accumu-

IFN- $\gamma$, TNF- $\alpha$ ) that act as recruiting signals for various proinflammatory cells are released to fight off viral infections [25]. The release of cytokines such as IFN- $\gamma$ is involved in the development of clinical symptoms such as fever, chills, headaches, dizziness, and fatigue. Other cytokines such as TNF- $\alpha$ can cause serious clinical conditions such as vascular leakage, cardiomyopathy, lung injury, acute-phase protein synthesis, and acute respiratory distress syndrome (ARDS) [28]. On the contrary, a recent editorial suggests that the role of the aforementioned cytokine storm is over exaggerated and that SARS-CoV-2 causes direct endothelial dysfunction and systemic inflammation leading to tachycardia, tachypnea, and hypotension [29]. Prior studies have shown that patients with COVID-19 despite not having high levels of cytokines, specifically IL-6, still have unfavorable outcomes with postmortem identification of significant alveolar microthrombi [30]. Despite the exact cause, it is clear lation and promotes tissue regeneration, anti-apoptosis and alveolar fluid clearance by producing extracellular vesicles, VEGF, HGF, and KGF. Abbreviations: ARDS acute respiratory distress syndrome, $A C E 2 R$ angiotensin-converting enzyme 2 receptor, $D C$ dendritic cells, $H G F$ hepatocyte growth factor, $I L$ interleukin, $I F N$ interferon, $I L-1 R A$ interlukin-1 receptor antagonist, $K G F$ keratinocyte growth factor, $M H C$ major histocompatibility complex, MSCs mesenchymal stem cells, PGE2 prostaglandin E2, SARS-CoV2 severe acute respiratory-associated coronavirus-2, $S O D-3$ superoxide dismutase, $S$-protein spike protein, $T N F-\alpha$ tumor necrosis factor alpha, TSG-6 tumor necrosis factor alpha stimulated gene-6, TGF- $\beta$ transforming growth factor-beta, TReg regulatory T cell, VEGF vascular endothelial growth factor

that inflammation and its effect on various organ systems whether induced by elevated levels of cytokines or directly from SARS-CoV-2 leads to the development of COVID-19. Ongoing research is needed to further elucidate the exact pathophysiological cause of COVID-19 lung injury.

\section{Acute respiratory distress syndrome (ARDS)}

The recent outbreak of SARS-CoV-2 has brought the world to an abrupt stop. The symptoms of the virus range from asymptomatic to mild upper respiratory tract infection to severe pneumonia [31]. About $67-85 \%$ of critically ill patients are known to develop ARDS making it one of the major reasons for the high mortality $(61.5 \%)$ rate among cases of SARS-CoV-2 [31, 32]. The pandemic has proven that the United States healthcare system needs improvement. 
With over $5 \%$ of cases needing ICU treatment, the pandemic rapidly outnumbered the available ICU beds and ventilator capacity across the United States causing a massive burden on our healthcare system [8]. Understanding the chronology from infection onset and controlling the later cascade of pro-inflammatory cytokines and chemokines that lead to ARDS, could lead to tremendous beneficial outcomes for the patients worldwide.

Although there are several clinical disorders associated with the development of ARDS, bacterial and viral pneumonia are the most common [33]. Normally, when infected either by bacteria or virus, the lungs trigger an immune response to clear the virus. However, ARDS is a result of hyperactivation of the innate immune system leading to rapid proliferation of $\mathrm{T}$ cells, macrophages and natural killer cells, and overproduction of over 100 inflammatory cytokines caused by SARS-CoV-2 infiltration [34]. Although the primary purpose of the inflammatory response is to clear the virus, it unfortunately has secondary adverse effects on the lungs [33]. One particular is an increased permeability of endothelial cells of the lungs caused by high levels of TNF$\alpha$, vascular endothelial growth factor (VEGF), and leukocyte signals in the lungs that destabilize the VE-cadherin bonds. These bonds usually maintain endothelial barrier integrity in the lung microvasculature [33]. Disruption of this barrier leads to alveolar edema. Similar to endothelial permeability, lung epithelial permeability is also compromised during an overactive immune response [33]. In this case, neutrophils disrupt intercellular junctions causing apoptosis and denudation that ultimately results in epithelial permeability [33]. During ARDS, the osmotic gradient that usually drives alveolar fluid clearance is also disrupted, which further exacerbates fluid retention in the alveoli [33].

With an increasing number of SARS-CoV-2 cases needing ICU treatment for ARDS and a limited number of beds and ventilators available, there is an increasing demand for prevention and treatment. Current treatments for COVID-19 are not definitive and lack evidence of safety and efficacy. Thus, new avenues for cellular repair following the development of ARDS should be investigated. UC and/or WJderived MSCs and their regenerative properties may have the potential to treat COVID-19 patients in the ICU suffering from ARDS, achieve complete recovery from SARS-CoV-2 symptoms, as well as reduce the burden on the healthcare system.

\section{Current treatment of COVID-19}

With the current status of the pandemic in mind, finding an effective and safe treatment has been the highest priority for research labs and pharmaceutical companies around the world [10]. At the moment, randomized controlled clinical trials have not shown evidence supporting prophylactic therapy or that any specific therapy (not including supportive therapy) improves outcomes in patients with COVID-19 [6]. Much of the current attention has been placed on treatments that have been previously used to treat SARS-CoV and MERS-CoV such as chloroquine/hydroxychloroquine, remdesivir, corticosteroids, and convalescent plasma [6].

Chloroquine and hydroxychloroquine seem to inhibit the viral entry into the host cells and decrease cytokine production and cell autophagy [6]. Notable side effects include hepatic failure, severe cutaneous reactions, and corrected QT interval prolongation especially with concurrent use of azithromycin or fluoroquinolones $[6,35]$. Remdesivir is an antiviral that is metabolized into an active $\mathrm{C}$-adenosine nucleoside triphosphate analogue inhibiting viral replication [6]. Currently, remdesivir has shown some efficacy in small clinical trials but it is not currently FDA-approved, and larger scale clinical trials are needed to further establish its efficacy [6]. Immunomodulation with corticosteroids in theory should help decrease the host hyper-immune response to the virus and decrease complications such as acute lung injury (ALI) and acute respiratory distress syndrome (ARDS) [6]. However, observational studies in patients infected with SARS and MERS, and treated with corticosteroids suggested that there was a delay in host viral clearance, increased rates of secondary infection and increased rates of complications such as psychosis, hyperglycemia, and avascular necrosis $[6,36,37]$. Convalescent plasma, plasma with antibodies from patients that have recovered from a specific infection, has been used effectively for salvage therapy in MERS and SARS patients [6, 38]. Convalescent plasma in theory could be efficacious when viremia is at its highest during the first 7-10 days of infection and contain SARS-CoV-2 antibodies. However, it is important to note that most commercial immunoglobulin preparations lack SARS-CoV-2 antibodies and more patients are required to recover from COVID-19 to have adequate plasma for large clinical trials [6].

Considering limited evidence to support the efficacy of aforementioned treatment modalities, associated adverse effects and the significant scientific constraints vaccines must undergo before successful clinical implementation, there is an increased need for quickly developing a safe and efficacious therapy [6]. UC and/or WJ-derived MSCs with their lack of severe adverse effects, effective immunomodulatory effects, regenerative capability and superiority over other biologics may be the answer for combating COVID-19 and the congruent healthcare burden that is currently present worldwide.

\section{Mesenchymal stem cells (MSCs)}

MSCs are non-hematopoietic progenitor cells first isolated from bone marrow by Friedenstein el al. [39] and originally called colony-forming unit fibroblasts. In 2006, the 
International Society for Cell Therapy defined human MSCs by the following criteria: plastic adhering when cultured; expressing surface markers CD105, CD73, and CD90; failing to express surface molecules CD45, CD34, CD14, CD11b, CD79alpha, CD19, and HLA-DR; and potential in vitro differentiation into osteoblasts, adipocytes, and chondrocytes [40]. Most recently, in 2017, Caplan pointed out that the primary function of MSCs is the paracrine secretion of various bioactive and immunomodulatory molecules acting as an in situ medication [41]. Along with the associated immunomodulatory effects of MSCs, they are also well known to decrease fibrosis, apoptosis, and induce tissue regeneration as well as produce significant amounts of EVs [45]. The regenerative and immunomodulatory capabilities of MSCs have made them an attractive treatment option in inflammatory and degenerative conditions [8]. Their efficacy and safety have been demonstrated and well documented from basic research and various clinical trials [12].

\section{MSCs and immunomodulation}

Allogenic MSCs express low levels of class II major histocompatibility complex (MHC), class I MHC and associated co-stimulatory molecules (B7-1, B7-2, CD80, CD86, and CD40) contributing to their immune privilege $[42,43]$. The therapeutic effects of MSCs can be partly attributed to their ability to secrete CKs, chemokines, GFs, angiogenic factors, and exosomes [7]. Immunomodulation by MSCs involves paracrine release of specific cytokines such as TGF- $\beta$, interleukin-1 receptor antagonist (IL-1RA), IL-10, IL-4, and IL-13 [7, 44]. These factors help to induce division arrest anergy, halting T cells at the G1 phase of the cell cycle, overall inhibiting not only T-cell proliferation but also B cells, natural killer cells and dendritic cells (DC) [46, 47]. MSCs paracrine signaling also modulates T-cell function by shifting the immune response from a Th1 and Th17 cell subset to a Treg and Th2 subset, decreasing levels of IL- 6 and TNF- $\alpha$ and reducing inflammation [48]. Along with the immuneregulatory modulation on adaptive immunity MSCs are also able to affect the innate immune response by influencing DC, macrophages, and neutrophils [49].

A study by Chen et al. utilized an in vitro model to explore the effect of human MSCs on the development of DC and found that MSCs can significantly modulate DC [50]. The study showed that due to the prostaglandin E2 production (PGE2) from MSCs, the differentiation of DC to the conventional DC phenotype was decreased and the differentiation into plasmacytoid DC increased, thereby weakening T-cell stimulation and shifting the T-cell population to a predominantly anti-inflammatory Th2 subset [50]. The modulation of adaptive immunity by MSCs was further characterized in a study by Dayan et al. in relation to macrophages and monocytes [51]. Levels of overall macrophages/monocytes were reduced with a reduction in pro-inflammatory M1 macrophages and an increase in "anti-inflammatory" M2 macrophages along with decreased levels of IL-1 $\beta$ and IL-6 [51].

It is well known that the neutrophil activation and subsequent release of reactive oxygen species (ROS), superoxide anion, peroxidases, and proteases are large contributors to the ARDS pathophysiology. In a study conducted by Jiang et al., the suppression of neutrophils by MSCs was characterized [49]. MSCs were shown to secrete SOD3, an antioxidant enzyme, which dampens the release of peroxidases and proteases and the overall oxidative burst of neutrophils [49]. MSCs were also able to directly engulf apoptotic neutrophils through ICAM-1 and inhibit the leakage of their toxic contents [49]. Another contributing factor is the tumor necrosis factor-inducible gene 6 protein released by MSCs which was shown to directly bind to IL-8 and chemokine CXCL8, inhibiting neutrophil migration, extravasation and activation $[52,53]$.

\section{MSC additional therapeutic benefits}

Along with MSC immunomodulatory functions, they have also been reported to promote tissue regeneration, inhibit fibrosis, aid in fluid clearance, and produce EVs [45, 54]. Additionally, intravenous infusions of MSCs are able to accumulate in the lung microvasculature as well as have an ability to directly hone and act on injured organs giving them great therapeutic potential $[53,55]$.

Production of growth factors such as keratinocyte growth factor (KGF), hepatocyte growth factor (HGF), and VEGF by MSCs has been shown to decrease collagen build up, decrease fibrosis and contribute to tissue regeneration [56, 57]. KGF stimulates type II alveolar epithelial cell proliferation, inhibits fibroblast proliferation and enhances the clearance of apoptotic cells by increasing matrix metalloproteinase-9, IL-1RA, and granulocyte-macrophage colony-stimulating factor [58, 59]. In a study by Aguilar et al., KGF was found to decrease collagen accumulation, decrease histological damage and induce endogenous type II pneumocyte proliferation in a bleomycin-induced pulmonary fibrosis model [58]. HGF increases tissue regeneration and cell survival as well as promotes suppression of fibrosis and chronic inflammation [60]. A study by Wang et al. [61] demonstrated that HGF from MSCs increased the expression of endothelial intercellular junction proteins, increased endothelial cell proliferation, and protected pulmonary microvascular endothelial cells. In a bleomycininduced lung injury model by Gazdhar et al. [62], HGF from induced pluripotent stem cells increased alveolar epithelial repair in vitro and improved lung fibrosis in vivo. MSCderived KGF and HGF have also been shown to stabilize Bcl-2, inhibit HIF1- $\alpha$ protein expression, and decrease ROS production, leading to an anti-apoptotic effect on the alveolar 
epithelial cells [63]. VEGF is an important growth factor that regulates vascular development and angiogenesis [64]. VEGF and HGF have been shown to stabilize the pulmonary endothelial barrier and restore pulmonary capillary permeability and inhibit cellular apoptosis [65].

MSCs are also capable of clearing alveolar fluid in the lungs, providing a great therapeutic benefit in patients with ARDS. Alveolar fluid movement is based on an osmotic gradient which is disrupted in ARDS leading to impaired alveolar fluid clearance (AFC) and higher rates of morbidity and mortality [54]. In a study by Loy et al. [66], human UC-derived MSCs were found to produce a large amount of angiopoietin-1, HGF and EVs restoring impaired AFC in an influenza A (H5N1) ALI model.

EVs are membrane-packed vesicles produced by MSCs and are formed by direct budding from the cell membrane and are about $40-1000 \mathrm{~nm}$ in size depending on the subclass $[67,68]$. There are several classes of EVs but exosomes and microvesicles (MVs) seem to be the most clinically relevant $[67,68]$. Exosomes are encapsulated in lipid bilayer membrane and contain annexins, tetraspanins, heat-shock proteins, transcription factors and genetic materials (mRNA, miRNA, DNA etc.) and are from an endosomal origin [67-69]. Microvesicles are formed by external budding of cell membranes and contain phosphatidylserine-containing proteins, cholesterol, sphingomyelin, ceramide, mRNAs, and microRNAs [67, 68]. In a study by Zhu et al. [70], MSC derived MVs were used as a treatment for E.coli endotoxininduced ALI, and the results showed that transfer of KGF mRNA from MVs was able to reduce neutrophil influx, decrease pulmonary edema and lower lung protein permeability. Exosomes have also been shown to have their own therapeutic applications. For example, a study by Dinh et al. [71] showed that exosomes were able to treat bleomycin and silica induced pulmonary fibrosis by inhibiting myofibroblast proliferation, decreasing collagen accumulation, and reestablishing normal alveolar structure.

\section{MSCs and COVID-19}

Although there are limited published clinical studies regarding MSC therapy in COVID-19, there is evidence of MSC as a therapy in ALI and ARDS that support the use of MSCs as a treatment in COVID-19. Several studies have shown that MSC therapy can decrease the overall mortality compared to non-MSC treated groups [72]. For example, in a study by Curley et al. [73], MSCs were shown to decrease lung inflammation, decrease histological lung injury and restore oxygenation and lung compliance in a ventilator-induced lung injury model. There are also several studies evaluating the use of MSCs in ARDS. ARDS is a major complication of COVID-19 and a major cause of ICU admissions and overall mortality in patients [30]. In a phase I, single-center, doubleblind, placebo-controlled clinical trial, ARDS patients were treated with allogenic MSCs [74]. No infusion toxicities or serious adverse effects related to MSCs administration were observed and levels of surfactant protein D (SPD) were significantly lower [74]. The lower SPD level has been reported to be associated with reduction in pulmonary inflammation [75]. In another investigation, the in vivo effects of MSCs were studied in two patients with severe ARDS by Simonson et al., with both patients receiving $2 \times 10^{6}$ cells/ $\mathrm{Kg}$ [76]. The two patients failed to improve initially after mechanical ventilation and extracorporeal ventilation [76]. After administration of MSCs, there was a decrease in the expression of markers for epithelial apoptosis and alveolar-capillary fluid leakage, as well as lower expression of proinflammatory cytokines and chemokines [76]. Overall, both patients improved with marked resolution in their hemodynamic instability, respiratory failure, and multiorgan failure [76].

Given that multiple studies of the use of MSCs in ALI and ARDS have shown evidence in regard to their safety and efficacy, there has been a growing demand for their use in patients with COVID-19. As of late, there has been several countries that have published data regarding the use of MSCs as a treatment for COVID-19. In a study done in Beijing YouAn Hospital, China, seven patients with COVID19 pneumonia were treated with MSCs [12]. After 2 days following MSC treatment the pulmonary function and symptoms of the all the seven patients drastically improved without any adverse effects. 3 of the patients recovered and were discharged 10 days after the treatment, with one of the patients being characterized as a "severe" case [12]. Within 3-6 days levels of $\mathrm{CD}^{+}{ }^{+} \mathrm{T}$ cells, $\mathrm{CD} 8^{+} \mathrm{T}$ cells, NK cells and TNF- $\alpha$ disappeared or decreased, with marked increases in regulatory DC and IL-10 compared to the placebo control group [12]. The gene expression of the MSCs showed that they were free of COVID-19 infection due to being $\mathrm{ACE}^{-}$and $\mathrm{TMPRSS}^{-}$, overall suggesting that MSCs were a safe and effective treatment for patients with COVID-19 [12]. In a clinical study done by Tang et al. [77] two patients with confirmed COVID-19 in Wuhan were treated with allogeneic menstrual blood-derived MSCs. The first patient was a 37-year-old woman with a medical history of hypertension and received IV infusions of MSCs on three consecutive days starting on February 5th, 2020 [77]. The patient's oxygen saturation $\left(\mathrm{SaO}_{2}\right)$ improved from a $98 \%$ on $100 \%$ fraction of inspired $\mathrm{O}_{2}\left(\mathrm{FiO}_{2}\right)$ to $97 \% \mathrm{SaO}_{2}$ on $55 \% \mathrm{FiO}_{2}$, and inflammation indicators (CRP, TNF- $\alpha$, IL-6) decreased [77]. The initial (Feb 1st and 4th) chest X-ray (CXR) findings of large, patchy and high density lesions in bilateral lung fields improved on Feb 6th and 10th with absorption of the exudate lesions in the bilateral lung fields. On the patient's followup visit on Feb 17th, the nucleic acid test for viral RNA was negative with resolution of initial symptoms (Fever and 
dyspnea) [77]. The second patient was a 71-year-old male, who also received three IV infusions of MSCs and had an almost identical clinical outcome to the first patient, with significant improvements in $\mathrm{SaO}_{2}$, inflammation indicators and CXR findings [77]. This study suggests that multiple infusions of MSCs may not only be safe, but also efficacious as a treatment for COVID-19. A very recent study published by Sanchez-Guijo et al. [78] on July 2020 supports the outcomes of the aforementioned studies. In this study, 13 patients who were previously treated with anti-inflammatory and antiviral therapies and that were under invasive mechanical ventilation due to COVID-19, were treated with allogenic MSCs [78]. All of the patients were administered their first dose of MSCs at a median of 7 days after mechanical ventilation initiation [78]. The median number of cells per dose was $0.98 \times 10^{6}$, with 10 of the 13 patients receiving two doses, 2 patients receiving a single dose and 1 patient receiving three doses. Overall clinical improvement was seen in $70 \%$ of the patients with none of the patients experiencing any adverse events related to the cell therapy. 7 of the patients were extubated and discharged, four patients remained intubated with two showing improvement in their ventilatory and radiological parameters and the remaining patients in stable condition [78].

With the supporting data of the use MSCs in ALI and ARDS and the early evidence seen in COVID-19 with similar treatments, a case for compassionate use of MSCs in COVID-19 patients can be made. However more randomized, controlled, multi-centered clinical trials are warranted to further establish the safety and efficacy of MSCs modality for its ultimate clinical utilization.

\section{Umbilical cord and Wharton's jelly}

MSCs and their specific attributes, including immunomodulation, tissue regeneration, fibrosis inhibition, fluid clearance, and ability to accumulate in the lung microvasculature have shown to be particularly valuable in the treatment of ALI, ARDS and COVID-19. With multiple sources of MSCs available for clinical use, the search for the most efficacious and safest source may end with the UC. The UC is a neonatal tissue composed of two arteries and a vein supported within connective tissue referred to as WJ, it connects the developing fetus and the placenta allowing the fetus to receive nutrient rich oxygenated blood from the placenta [79]. WJ is a mucoid connective tissue between the amniotic epithelium and the umbilical vessels within the UC, and provides protection, cushion, and structural support to the umbilical vessels [19]. The UC can be obtained without having a patient undergo an invasive procedure, unlike sources like DP, BM, and adipose tissue. The UC and the WJ within it, are also both considered an after-birth tissue and normally discarded as medical waste, thus contributing more to their accessibility [80]. UC-MSCs can be easily and quickly expanded, due to their doubling times, to reach the numbers needed to have a clinically efficacious treatment [81]. WJ is rich with MSCs, GFs, CKs, and EVs especially compared to other sources, with the highest concentration of MSCs per milliliter than any other allogenic tissue [19, 82]. UC-MSCs have been shown to be significant regulators of activated $\mathrm{T}$ cells contributing to their superb ability for immunosuppression and regulation of the innate and adaptive immune responses. The UC has been the most widely used first choice for allogenic MSCs in many immunocompromised states [83].

\section{UC and/or WJ and COVID-19: current evidence}

UC and/or WJ-derived MSCs have been used in multiple clinical situations and have shown to be safe when appropriate cell concentrations, dosages, and infusion rates are used [84]. In a case study reported in China by Liang et al. [85], a patient with COVID-19 was treated with a triple IV infusion of UC-derived MSCs. The patient was a 65-yearold female who presented on day 1 with a fever of $38.2^{\circ} \mathrm{C}$, chest tightness and a $\mathrm{SPO}_{2}$ of $81 \%$ along with a positive real-time reverse transcription polymerase chain reaction (RT-PCR) assay for SARS-CoV-2 [85]. On day 12 after she was diagnosed as a critically ill-type COVID-19, with multiorgan injury, ARDS and severe pneumonia, and treatment with UC-MSCs was discussed and approved [85]. Allogenic UC-MSCs were IV administered on days 13, 16, and 19 [85]. After the first infusion, no obvious adverse effects were noted, and serum CRP, ALT and AST levels diminished along with improvements in the vitals [85]. Following the second infusion, WBC count and neutrophil levels decreased to a normal range and $\mathrm{CD} 3^{+} \mathrm{T}$ cell, $\mathrm{CD} 4^{+} \mathrm{T}$ cell, and $\mathrm{CD} 8^{+} \mathrm{T}$ counts were increased, and the patient had their tracheal cannula removed and was ambulating by day 17 [85]. On day 21, the patient was transferred out of the ICU with CT images showing resolution of initial ground glass opacities, along with near normal vitals, laboratory values and was discharged from the hospital on day 30 [85]. In another single-center open label, individually randomized standard treatment-controlled trial conducted in Hubei Province China, UC-MSCs were assessed as a treatment of COVID-19 [86]. A total of 41 patients diagnosed with COVID-19 were enrolled in the study and divided into 2 groups: a standard treatment group (control group) and a standard treatment group (treatment group) with the addition of UC-MSCs [86]. The standard treatment included supplemental oxygen (invasive ventilation or non-invasive), antibiotics (oral moxifloxacin and others depending on bacterial sensitivity), antivirals (abidor/oseltamivir) and glucocorticoids [86]. 12 patients were enrolled in the treatment group and 29 patients were enrolled in the stand alone 


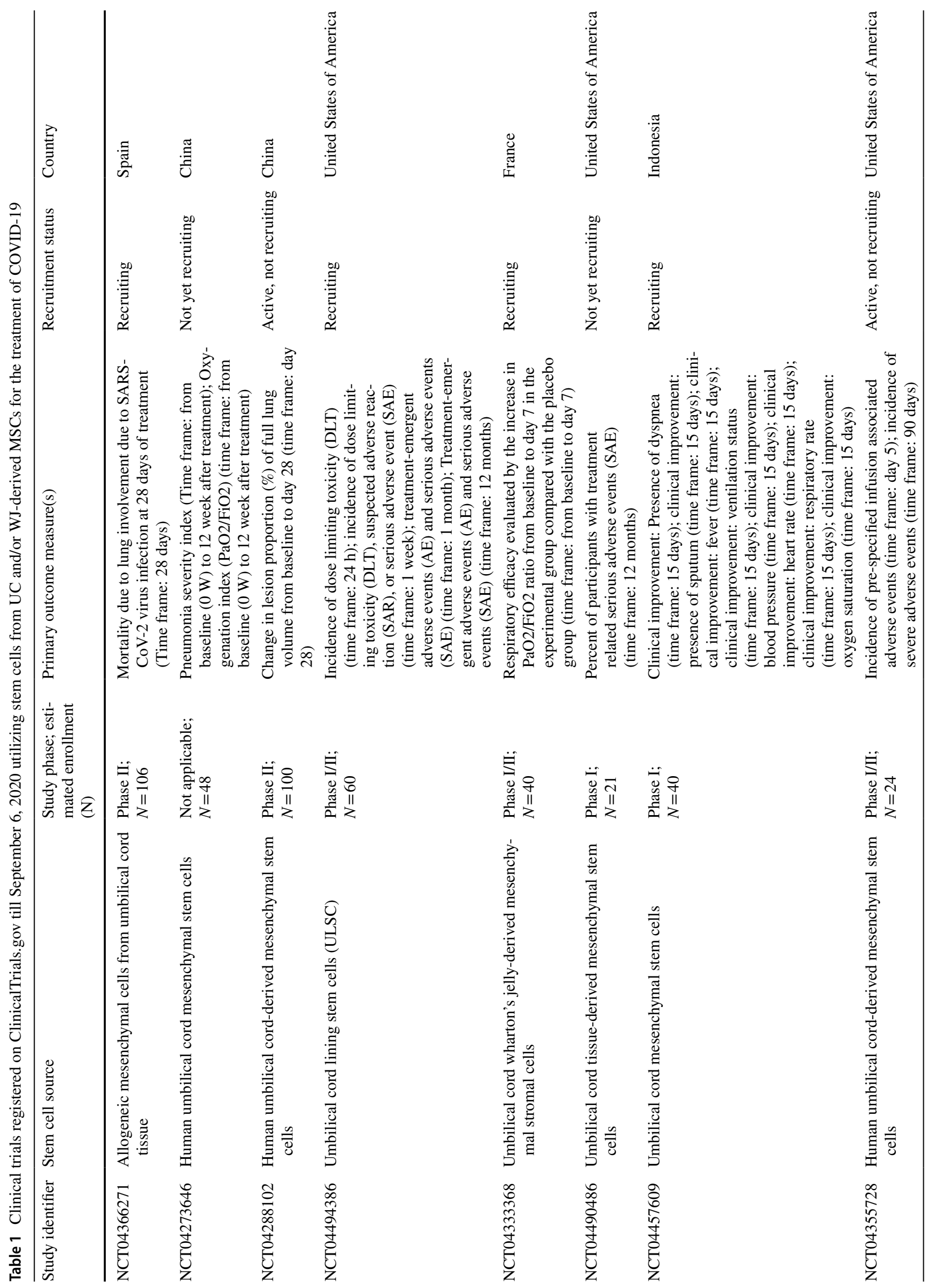




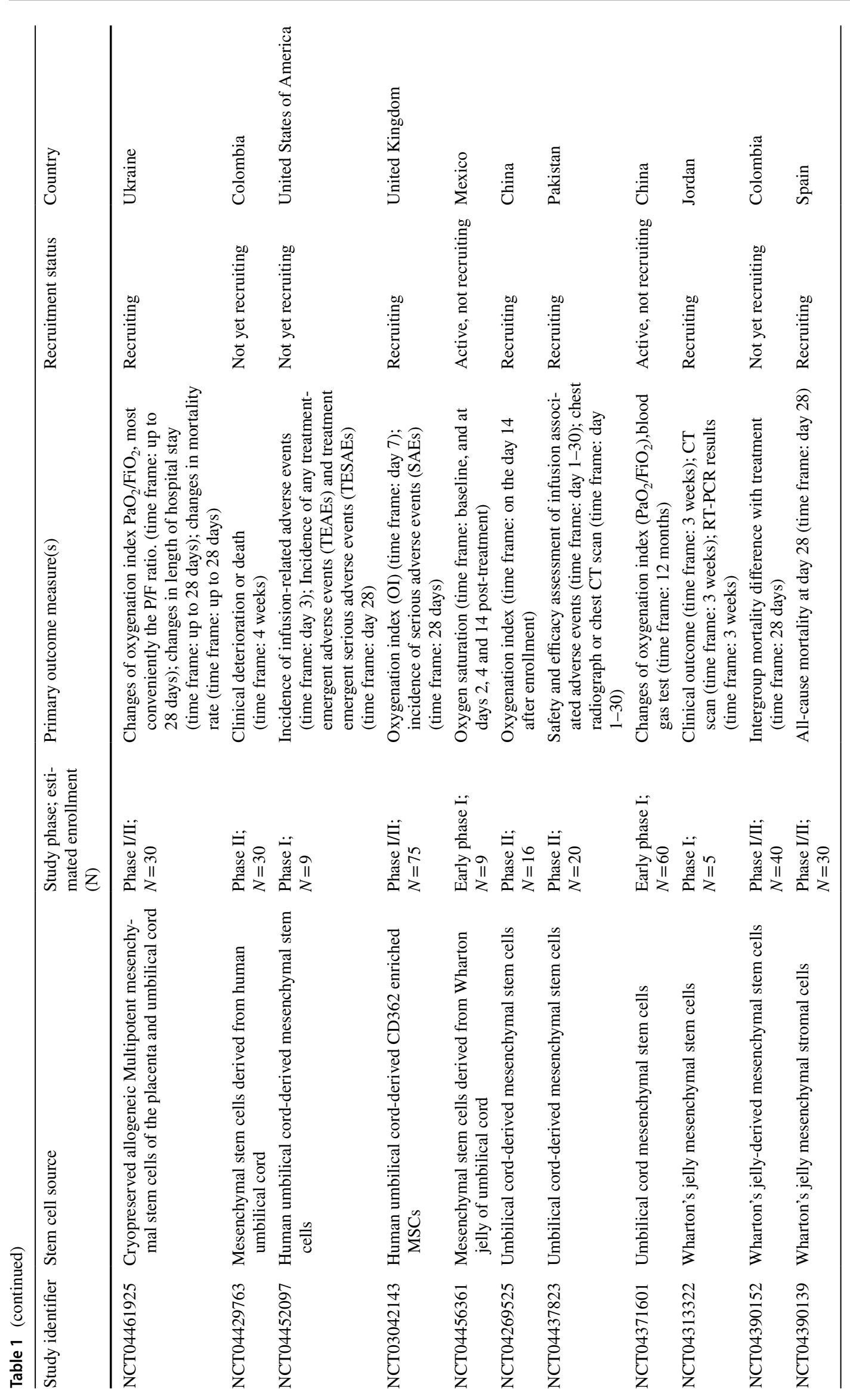


standard treatment group with no significant differences in demographics, laboratory test results, clinical symptoms and conditions [86]. All of the patients in the treatment group did not have to undergo invasive ventilation and were all discharged, with a 28-days mortality rate of $0 \%$ [86]. Out of the 29 patients in the control group, 4 progressed to critical illness and had to receive invasive ventilation and the group had a 28 -days morality rate of $10.34 \%$ [86]. Symptomatic and clinical improvement was seen in more patients and at a faster rate in the treatment group with no noted adverse effects [86]. The effects of MSCs were noted by the decrease in CRP and IL-6 along with improvements in arterial blood gas, lymphocyte counts and the patient's chest CTs compared to the control group [86].

Recently, data from a phase I clinical trial involving UCMSCs in patients with COVID-19 were published that further support previous results [87]. A total of 18 patients with moderate to severe COVID-19 were enrolled in a parallel assigned, controlled, non-randomized, phase I clinical trial [87]. The 18 patients were evenly divided into 2 groups both of which received standard COVID-19 treatment regimens with the treatment group ( 9 patients) receiving 3 cycles of IV UC-MSCs [87]. Other than temporary facial flushing seen in two patients and transient fever in three patients that spontaneously resolved within $24 \mathrm{~h}$, there were no serious adverse events associated with UC-MSCs [87]. Invasive ventilation was required for only one patient in the treatment group compared to four patients in the control group, along with complete resolution of pathological lung changes seen on CT in the treatment group [87]. Levels of IFN- $\gamma$, TNF- $\alpha$, IL-6, and IL-1 receptor were all found to be reduced within 14 days of UC-MSC infusion [87]. With this phase I trial showing that infusion of UC-MSCs is safe and tolerable in patients with COVID-19 along with signs of promising efficacy, a phase II clinical trial (NCT04288102) was proposed and will be conducted to further assess the efficacy, as also indicated in Table 1 [87].

\section{Ongoing trials}

Several pre-clinical and clinical studies have investigated the potential of MSCs including UC and/or WJ-derived MSCs for treating COVID-19, including management of associated cytokine storm. Though the results are encouraging, the limited literature still warrants more studies to establish safety and efficacy of UC and/or WJ-derived MSCs to treat and manage symptoms associated with the COVID-19 infection. Eventually, multi-center, controlled, randomized trials will be needed to adequately assess the future of these MSCs in the treatment of COVID-19. As of September 6, 2020, there are 19 on-going studies related to use of UC and/or WJ-derived MSCs (Table 1) registered on ClinicalTrials.gov.

\section{Conclusion}

Millions of people have been affected by COVID-19 and the ripple effects of this pandemic is yet to be discovered. The scientific and medical community is desperate to find a way to combat COVID-19 and diminished any further damage. With no definitive treatment not yet available, MSCs seem to be a promising answer to the current pandemic. The case for the use MSCs on a compassionate basis can be made due to their ability to decrease inflammation and repair endogenous tissues. Much of the current evidence points to the UC as most promising source of MSCs. Although more preclinical and clinical studies are needed, allogenic UC-MSCs and/ or WJ-MSCS have evidence of being an available, safe and effective option to combat COVID-19 and the congruent strain on medical and hospital resources.

\section{Compliance with ethical standards}

Conflict of interest The authors declare that they have no conflict of interest.

\section{References}

1. Fung TS, Liu DX. Human Coronavirus: host-pathogen interaction. Annu Rev Microbiol. 2019;73:529-57.

2. Guo L, Yu H, Gu W, Luo X, Li R, Zhang J, et al. Autophagy negatively regulates transmissible gastroenteritis virus replication. Sci Rep. 2016;6:23864.

3. Bulut C, Kato Y. Epidemiology of COVID-19. Turk J Med Sci. 2020;50(Si-1):563-70.

4. COVID-19 Dashboard by the Center for Systems Science and Engineering (CSSE) at Johns Hopkins University (JHU). Available from: https://coronavirus.jhu.edu/map.html. Accessed 6 Sept 2020.

5. Brown BL, McCullough J. Treatment for emerging viruses: convalescent plasma and COVID-19. Transfus Apher Sci. 2020;59(3):102790.

6. Sanders JM, Monogue ML, Jodlowski TZ, Cutrell JB. Pharmacologic treatments for coronavirus disease 2019 (COVID-19): a review. JAMA. 2020. https://doi.org/10.1001/jama.2020.601.

7. Rogers CJ, Harman RJ, Bunnell BA, Schreiber MA, Xiang C, Wang FS, et al. Rationale for the clinical use of adipose-derived mesenchymal stem cells for COVID-19 patients. J Transl Med. 2020;18(1):203.

8. Gupta A, Kashte S, Gupta M, Rodriguez HC, Gautam SS, Kadam S. Mesenchymal stem cells and exosome therapy for COVID-19: current status and future perspective. Hum Cell. 2020;33(4):907-18.

9. Harrell CR, Sadikot R, Pascual J, Fellabaum C, Jankovic MG, Jovicic N, et al. Mesenchymal stem cell-based therapy of inflammatory lung diseases: current understanding and future perspectives. Stem Cells Int. 2019;2019:4236973.

10. Atluri S, Manchikanti L, Hirsch JA. Expanded umbilical cord mesenchymal stem cells (UC-MSCs) as a therapeutic strategy in 
managing critically Ill COVID-19 patients: the case for compassionate use. Pain Physician. 2020;23(2):E71-e83.

11. Tsuchiya A, Takeuchi S, Iwasawa T, Kumagai M, Sato T, Motegi S, et al. Therapeutic potential of mesenchymal stem cells and their exosomes in severe novel coronavirus disease 2019 (COVID-19) cases. Inflamm Regen. 2020;22(40):14.

12. Leng Z, Zhu R, Hou W, Feng Y, Yang Y, Han Q, et al. Transplantation of ACE2(-) mesenchymal stem cells improves the outcome of patients with COVID-19 pneumonia. Aging Dis. 2020;11(2):216-28.

13. Fabre H, Ducret M, Degoul O, Rodriguez J, Perrier-Groult E, Aubert-Foucher E, et al. Characterization of different sources of human MSCs expanded in serum-free conditions with quantification of chondrogenic induction in 3D. Stem Cells Int. 2019;2019:2186728.

14. Potty AGR, Gupta A, Rodriguez HC, Stone IW, Maffulli N. Intraosseous bioplasty for a subchondral cyst in the lateral condyle of femur. J Clin Med. 2020;9(5):1358.

15. Mohamed-Ahmed S, Fristad I, Lie SA, Suliman S, Mustafa K, Vindenes H, et al. Adipose-derived and bone marrow mesenchymal stem cells: a donor-matched comparison. Stem Cell Res Ther. 2018;9(1): 168

16. Usuelli FG, D'Ambrosi R, Maccario C, Indino C, Manzi L, Maffulli N. Adipose-derived stem cells in orthopaedic pathologies. Br Med Bull. 2017;124(1):31-54.

17. Ravindran S, Huang CC, George A. Extracellular matrix of dental pulp stem cells: applications in pulp tissue engineering using somatic MSCs. Front Physiol. 2014;4:395.

18. Jeschke MG, Gauglitz GG, Phan TT, Herndon DN, Kita K. Umbilical cord lining membrane and Wharton's jelly-derived mesenchymal stem cells: the similarities and differences. Open Tissue Eng Regener Med J. 2011;4(1):21-7.

19. Gupta A, El-Amin SF 3rd, Levy HJ, Sze-Tu R, Ibim SE, Maffulli N. Umbilical cord-derived Wharton's jelly for regenerative medicine applications. J Orthop Surg Res. 2020;15(1):49.

20. Carlin R, Davis D, Weiss M, Schultz B, Troyer D. Expression of early transcription factors Oct- 4 , Sox- 2 and Nonog by porcine umbilical cord (PUC) matrix cells. Reprod Biol Endocrinol. 2006;4(1):8.

21. La Rocca G, Anzalone R, Corrao S, Magno F, Loria T, Lo Iacono $\mathrm{M}$, et al. Isolation and characterization of Oct $-4^{+} / \mathrm{HLA}-\mathrm{G}^{+}$ mesenchymal stem cells from human umbilical cord matrix: differentiation potential and detection of new markers. Histochem Cell Biol. 2009;131(2):267-82.

22. Channappanavar R, Zhao J, Perlman S. T cell-mediated immune response to respiratory coronaviruses. Immunol Res. 2014;59(1-3):118-28.

23. Stawicki SP, Jeanmonod R, Miller AC, et al. The 2019-2020 Novel Coronavirus (Severe Acute Respiratory Syndrome Coronavirus 2) pandemic: a joint American College of Academic International Medicine-World Academic Council of emergency medicine multidisciplinary COVID-19 Working Group Consensus Paper. J Glob Infect Dis. 2020;12(2):47-93.

24. Li W, Moore MJ, Vasilieva N, et al. Angiotensin-converting enzyme 2 is a functional receptor for the SARS coronavirus. Nature. 2003;426(6965):450-4.

25. Machhi J, Herskovitz J, Senan AM, et al. The natural history, pathobiology, and clinical manifestations of SARS-CoV-2 infections. J Neuroimmune Pharmacol. 2020. https://doi. org/10.1007/s11481-020-09944-5.

26. Wang D, Hu B, Hu C, et al. Clinical characteristics of 138 hospitalized patients with 2019 novel coronavirus-infected Pneumonia in Wuhan, China. JAMA. 2020;323(11):1061-9.

27. Stone IW, Gupta A, Rodriguez HC, Mistovich RJ, Gupta M, Potty AG, et al. Prone Anesthesia for a super-super morbidly obese patient undergoing neurosurgery: a case report. OBM
Neurobiol. 2020;4(2):7. https://doi.org/10.21926/obm.neuro biol.2002064.

28. Shimabukuro-Vornhagen A, Gödel P, Subklewe M, et al. Cytokine release syndrome. J Immunother Cancer. 2018;6(1):56

29. Sinha P, Matthay MA, Calfee CS. Is a "Cytokine Storm" relevant to COVID-19? JAMA. 2020. https://doi.org/10.1001/jamai nternmed.2020.3313.

30. Ackermann M, Verleden SE, Kuehnel M, Haverich A, Welte $\mathrm{T}$, Laenger $\mathrm{F}$, et al. Pulmonary vascular endothelialitis, thrombosis, and angiogenesis in COVID-19. N Engl J Med. 2020;383(2):120-8.

31. Xiao K, Hou F, Huang X, Li B, Qian ZR, Xie L. Mesenchymal stem cells: current clinical progress in ARDS and COVID-19. Stem Cell Res Ther. 2020;11(1):305.

32. Huang C, Wang Y, Li X, et al. Clinical features of patients infected with 2019 novel coronavirus in Wuhan. China Lancet. 2020;395(10223):497-506.

33. Huppert LA, Matthay MA, Ware LB. Pathogenesis of acute respiratory distress syndrome. Semin Respir Crit Care Med. 2019;40(1):31-9.

34. Sun X, Wang T, Cai D, et al. Cytokine storm intervention in the early stages of COVID-19 pneumonia. Cytokine Growth Factor Rev. 2020;53:38-42.

35. Ferner R, Aronson JK. Choroquine and hydroxychloroquine in COVID-19. BMJ. 2020;8(369):m1432.

36. Russell CD, Millar JE, Baillie JK. Clinical evidence does not support corticosteroid treatment for 2019-nCoV lung injury. Lancet. 2020;395(10223):473-5.

37. Arabi YM, Mandourah Y, Al-Hameed F, Sindi AA, Almekhlafi GA, Hussein MA, et al. Corticosteroid therapy for critically Ill patients with middle east respiratory syndrome. Am J Respir Crit Care Med. 2018;197(6):757-67.

38. Mair-Jenkins J, Saavedra-Campos M, Baillie JK, Cleary P, Khaw FM, Lim WS, et al. The effectiveness of convalescent plasma and hyperimmune immunoglobulin for the treatment of severe acute respiratory infections of viral etiology: a systematic review and exploratory meta-analysis. J Infect Dis. 2015;211(1):80-90.

39. Friedenstein AJ, Petrakova KV, Kurolesova AI, Frolova GP. Heterotopic of bone marrow. Analysis of precursor cells for osteogenic and hematopoietic tissues. Transplantation. 1968;6(2):230-47.

40. Dominici M, Le Blanc K, Mueller I, Slaper-Cortenbach I, Marini F, Krause D, et al. Minimal criteria for defining multipotent mesenchymal stromal cells. The International Society for Cellular Therapy position statement. Cytotherapy. 2006;8(4):315-7.

41. Zhou Y, Yamamoto Y, Xiao Z, Ochiya T. The Immunomodulatory functions of mesenchymal stromal/stem cells mediated via paracrine activity. J Clin Med. 2019;7:1025.

42. De Miguel MP, Fuentes-Julián S, Blázquez-Martínez A, Pascual CY, Aller MA, Arias J, Arnalich-Montiel F. Immunosuppressive properties of mesenchymal stem cells: advances and applications. Curr Mol Med. 2012;12(5):574-91.

43. Lee JW, Gupta N, Serikov V, Matthay MA. Potential application of mesenchymal stem cells in acute lung injury. Expert Opin Biol Ther. 2009;9(10):1259-70.

44. Maacha S, Sidahmed H, Jacob S, Gentilcore G, Calzone R, Grivel $\mathrm{JC}$, et al. Paracrine mechanisms of mesenchymal stromal cells in angiogenesis. Stem Cells Int. 2020;9(2020):4356359.

45. Medhat D, Rodriguez CI, Infante A. Immunomodulatory effects of MSCs in bone healing. Int J Mol Sci. 2019;20(21):E5467.

46. Glennie S, Soeiro I, Dyson PJ, Lam EW, Dazzi F. Bone marrow mesenchymal stem cells induce division arrest anergy of activated T cells. Blood. 2005;105(7):2821-7.

47. De Miguel MP, Fuentes-Julián S, Blázquez-Martínez A, Pascual CY, Aller MA, Arias J, et al. Immunosuppressive properties of 
mesenchymal stem cells: advances and applications. Curr Mol Med. 2012;12(5):574-91.

48. Duffy MM, Ritter T, Ceredig R, Griffin MD. Mesenchymal stem cell effects on T-cell effector pathways. Stem Cell Res Ther. 2011;2(4):34.

49. Jiang D, Muschhammer J, Qi Y, Kügler A, de Vries JC, Saffarzadeh M, et al. Suppression of neutrophil-mediated tissue damage - a novel skill of mesenchymal stem cells. Stem Cells. 2016;34(9):2393-406.

50. Chen L, Zhang W, Yue H, Han Q, Chen B, Shi M, et al. Effects of human mesenchymal stem cells on the differentiation of dendritic cells from CD34 ${ }^{+}$cells. Stem Cells Dev. 2007;16(5):719-31.

51. Dayan V, Yannarelli G, Billia F, Filomeno P, Wang XH, Davies JE, et al. Mesenchymal stromal cells mediate a switch to alternatively activated monocytes/macrophages after acute myocardial infarction. Basic Res Cardiol. 2011;106(6):1299-310.

52. Dyer DP, Thomson JM, Hermant A, Jowitt TA, Handel TM, Proudfoot AE, et al. TSG-6 inhibits neutrophil migration via direct interaction with the chemokine CXCL8. J Immunol. 2014;192(5):2177-85.

53. Monsel A, Zhu YG, Gennai S, Hao Q, Liu J, Lee JW. Cell-based therapy for acute organ injury: preclinical evidence and ongoing clinical trials using mesenchymal stem cells. Anesthesiology. 2014;121(5):1099-121.

54. Ware LB, Matthay MA. Alveolar fluid clearance is impaired in the majority of patients with acute lung injury and the acute respiratory distress syndrome. Am J Respir Crit Care Med. 2001;163(6):1376-83

55. Lee RH, Pulin AA, Seo MJ, Kota DJ, Ylostalo J, Larson BL, et al. Intravenous hMSCs improve myocardial infarction in mice because cells embolized in lung are activated to secrete the antiinflammatory protein TSG-6. Cell Stem Cell. 2009;5(1):54-63.

56. Li X, Yue S, Luo Z. Mesenchymal stem cells in idiopathic pulmonary fibrosis. Oncotarget. 2017;8(60):102600-166.

57. Behnke J, Kremer S, Shahzad T, Chao CM, Böttcher-Friebertshäuser E, Morty RE, et al. MSC based therapies-new perspectives for the injured lung. J Clin Med. 2020;9(3):E682.

58. Aguilar S, Scotton CJ, McNulty K, Nye E, Stamp G, Laurent G, et al. Bone marrow stem cells expressing keratinocyte growth factor via an inducible lentivirus protects against bleomycin-induced pulmonary fibrosis. PLoS ONE. 2009;4(11):e8013.

59. Shyamsundar M, McAuley DF, Ingram RJ, Gibson DS, O'Kane $\mathrm{D}$, McKeown ST, et al. Keratinocyte growth factor promotes epithelial survival and resolution in a human model of lung injury. Am J Respir Crit Care Med. 2014;189(12):1520-9.

60. Nakamura T, Sakai K, Nakamura T, Matsumoto K. Hepatocyte growth factor twenty years on: much more than a growth factor. $\mathrm{J}$ Gastroenterol Hepatol. 2011;26(Suppl 1):188-202.

61. Wang H, Zheng R, Chen Q, Shao J, Yu J, Hu S. Mesenchymal stem cells microvesicles stabilize endothelial barrier function partly mediated by hepatocyte growth factor (HGF). Stem Cell Res Ther. 2017;8(1):211.

62. Gazdhar A, Grad I, Tamò L, Gugger M, Feki A, Geiser T. The secretome of induced pluripotent stem cells reduces lung fibrosis in part by hepatocyte growth factor. Stem Cell Res Ther. 2014;5(6): 123

63. Bernard O, Jeny F, Uzunhan Y, Dondi E, Terfous R, Label R, et al. Mesenchymal stem cells reduce hypoxia-induced apoptosis in alveolar epithelial cells by modulating HIF and ROS hypoxic signaling. Am J Physiol Lung Cell Mol Physiol. 2018;314(3):L360-L371371.

64. Hu K, Olsen BR. The roles of vascular endothelial growth factor in bone repair and regeneration. Bone. 2016;10(91):30-8.

65. Yang Y, Chen QH, Liu AR, Xu XP, Han JB, Qiu HB. Synergism of MSC-secreted HGF and VEGF in stabilising endothelial barrier function upon lipopolysaccharide stimulation via the Rac1 pathway. Stem Cell Res Ther. 2015;16(6):250.

66. Loy H, Kuok DIT, Hui KPY, Choi MHL, Yuen W, Nicholls JM, et al. Therapeutic implications of human umbilical cord mesenchymal stromal cells in attenuating influenza $\mathrm{A}(\mathrm{H} 5 \mathrm{~N} 1)$ virusassociated acute lung injury. J Infect Dis. 2019;219(2):186-96.

67. Raposo G, Stoorvogel W. Extracellular vesicles: exosomes, microvesicles, and friends. J Cell Biol. 2013;200(4):373-83.

68. Konala VB, Mamidi MK, Bhonde R, Das AK, Pochampally R, Pal R. The current landscape of the mesenchymal stromal cell secretome: a new paradigm for cell-free regeneration. Cytotherapy. 2016;18(1):13-24.

69. Keshtkar S, Azarpira N, Ghahremani MH. Mesenchymal stem cell-derived extracellular vesicles: novel frontiers in regenerative medicine. Stem Cell Res Ther. 2018;9(1):63.

70. Zhu YG, Feng XM, Abbott J, Fang XH, Hao Q, Monsel A, et al. Human mesenchymal stem cell microvesicles for treatment of Escherichia coli endotoxin-induced acute lung injury in mice. Stem Cells. 2014;32(1):116-25.

71. Dinh PC, Paudel D, Brochu H, Popowski KD, Gracieux MC, Cores $\mathrm{J}$, et al. Inhalation of lung spheroid cell secretome and exosomes promotes lung repair in pulmonary fibrosis. Nat Commun. 2020;11(1):1064.

72. McIntyre LA, Moher D, Fergusson DA, Sullivan KJ, Mei SH, Lalu M, et al. Efficacy of mesenchymal stromal cell therapy for acute lung injury in preclinical animal models: a systematic review. PLoS ONE. 2016;11(1):e0147170.

73. Curley GF, Hayes M, Ansari B, Shaw G, Ryan A, Barry F, et al. Mesenchymal stem cells enhance recovery and repair following ventilator-induced lung injury in the rat. Thorax. 2012;67(6):496-501.

74. Zheng G, Huang L, Tong H, Shu Q, Hu Y, Ge M, et al. Treatment of acute respiratory distress syndrome with allogeneic adiposederived mesenchymal stem cells: a randomized, placebo-controlled pilot study. Respir Res. 2014;4(15):39.

75. Mackay RA, Townsend JP, Calvert J, Anthony M, Wilkinson $\mathrm{AR}$, Postle AD, et al. Increased surfactant protein-D levels in the airways of preterm neonates with sepsis indicated responses to infectious challenges. Acta Paediatr. 2019;108(5):870-6.

76. Simonson OE, Mougiakakos D, Heldring N, Bassi G, Johansson HJ, Dalén M, et al. In vivo effects of mesenchymal stromal cells in two patients with severe acute respiratory distress syndrome. Stem Cells Transl Med. 2015;4(10):1199-213.

77. Tang L, Jiang Y, Zhu M, Chen L, Zhou X, Zhou C, et al. Clinical study using mesenchymal stem cells for the treatment of patients with severe COVID-19. Front Med. 2020;6:1-10.

78. Sánchez-Guijo F, García-Arranz M, López-Parra M, Monedero P, Mata-Martínez C, Santos A, et al. Adipose-derived mesenchymal stromal cells for the treatment of patients with severe SARSCoV-2 pneumonia requiring mechanical ventilation. A proof of concept study. EClin Med. 2020;25:100454.

79. Goplerud JM, Delivoria-Papadopoulos M. Physiology of the placenta-gas exchange. Ann Clin Lab Sci. 1985;15(4):270-8.

80. El Omar R, Beroud J, Stoltz JF, Menu P, Velot E, Decot V. Umbilical cord mesenchymal stem cells: the new gold standard for mesenchymal stem cell-based therapies. Tissue Eng Part B Rev. 2014;20(5):523-44.

81. Weiss ML, Troyer DL. Stem cells in the umbilical cord. Stem Cell Rev. 2006;2(2):155-62.

82. Arutyunyan I, Elchaninov A, Makarov A, Fatkhudinov T. Umbilical cord as prospective source for mesenchymal stem cell-based therapy. Stem Cells Int. 2016;2016:6901286.

83. Can A, Celikkan FT, Cinar O. Umbilical cord mesenchymal stromal cell transplantations: a systemic analysis of clinical trials. Cytotherapy. 2017;19(12):1351-82. 
84. Bauer G, Elsallab M, Abou-El-Enein M. Concise review: a comprehensive analysis of reported adverse events in patients receiving unproven stem cell-based interventions. Stem Cells Transl Med. 2018;7(9):676-85.

85. Liang B, Chen J, Li T, Wu H, Yang W, Li Y, et al. Clinical remission of a critically ill COVID-19 patient treated by human umbilical cord mesenchymal stem cells: a case report. Medicine (Baltimore). 2020;99(31):e21429.

86. Shu L, Niu C, Li R, Huang T, Wang Y, Huang M, et al. Treatment of severe COVID-19 with human umbilical cord mesenchymal stem cells. Stem Cell Res Ther. 2020;11(1):361.
87. Meng F, Xu R, Wang S, Xu Z, Zhang C, Li Y, et al. Human umbilical cord-derived mesenchymal stem cell therapy in patients with COVID-19: a phase 1 clinical trial. Signal Transduct Target Ther. 2020;5(1):172.

Publisher's Note Springer Nature remains neutral with regard to jurisdictional claims in published maps and institutional affiliations. 